\title{
A symplectic integrator for dynamic coupling between nonlinear vessel motion with variable cross-section and bottom topography and interior shallow-water sloshing
}

\author{
Hamid Alemi Ardakani \\ Department of Mathematics, University of Surrey, Guildford, Surrey GU2 7XH, UK \\ Email: alemi.hamid@gmail.com
}

- Abstract -

The coupled motion between shallow-water sloshing in a moving vessel with variable cross-section and bottom topography, and the vessel dynamics is considered, with the vessel dynamics restricted to horizontal motion governed by a nonlinear spring. The coupled fluid and vessel equations in Eulerian coordinates are transformed to the Lagrangian particle path setting which leads to a formulation with nice properties for numerical simulation. In the Lagrangian representation, a simple and fast numerical algorithm based on the Störmer-Verlet method, is implemented. The numerical scheme conserves the total energy in the system, as well as giving the partition of energy between the fluid and vessel. Numerical simulations of the coupled nonlinear dynamics are presented.

\section{Introduction}

The effect of liquid sloshing on the dynamics and control of liquid transport, e.g. the terrestrial transport of liquids, oil and liquid natural gas in ships, and fuel in aviation and astrodynamics, has motivated a wide range of research. An indication of the breadth of research in this area is the books of IBRAHIm [20] and FALTINSEN AND TIMOKHA [12] and references therein. The coupled dynamics between fluid sloshing and vessel motion brings in a new dimension and the potential for enhancing or diminishing the sloshing motion through vessel dynamics. The coupled problem is of great practical interest in the transport of liquids along roads, maritime fluid transport, and industrial applications. Examples of where dynamic coupling is of interest is the sloshing of water on the the deck of fishing vessels $[10,6,1]$, transport of liquid by robots [36, 37], motion planning for industrial control [17, 29, 13, 11], sloshing in automobile fuel tanks [42], in tuned liquid dampers (TLDs) [21, 14], and motion of water waves in a suspended container [8]. The book of MOISEYEV AND RUMYANTSEV [26] covers general aspects of the dynamic coupling between vessel motion and interior fluid motion. 
Analysis and experiment are carried out in [8] on a horizontal rectangular wave tank which swings at the lower end of a pendulum such that the base of the tank remains horizontal. The linearized form of the shallow-water equations are solved coupled to the container equation with linear coupling. A transcendental relation is derived for the periodic solutions of the coupled system, and a curious resonance where the second mode of the coupled problem appeares to resonate with the natural frequency of the vessel is found. This behaviour could not be explained as the resonant solution did not satisfy the characteristic equation. The exact equations for the Cooker's sloshing experiment are derived in [2] with a variational principle. In [3] the coupled dynamics between shallow-water sloshing and horizontal vehicle motion is considered. In [3] a nonlinear shallow-water model is coupled to a linear vessel equation. Hamiltonian formulations are derived for the fully linear and nonlinear coupled systems and symplectic integrators with excellent energy conservation over long times are developed. A linear model for finite depth fluid is studied in [43] but a mechanism for continued energy input to support a resonant behaviour is not found. This problem is resolved in [4] where a highly nonlinear transcendental characteristic equation for the natural frequencies of the coupled motion is derived in the finite depth problem. Two derivations of the characteristic equation are given, one based on a cosine expansion and the other based on a class of vertical eigenfunctions and it is proved that the characteristic equations are equivalent. The most important observation in [4] is the discovery of an internal $1: 1$ resonance in the fully two-dimensional finite depth model where the frequency of one of the symmetric sloshing modes is equal to the frequency of the anti-symmetric modes and the vessel. In [34] the dynamic coupling problem is investigated for Cooker's sloshing experiment with baffles and it is found that a multitude of resonance situations can occur in the system, from $1: 1$ resonances to multifold $1: 1: \cdots: 1$ resonances. In $[40,41]$ the shallowwater theory of Cooker [8] is extended to a multi-compartment rectangular vessel and some experimental results are reported. In [33] the nonlinear energy transfer between fluid sloshing and vessel motion is studied through derivation of a nonlinear normal form close to an internal $1: 1$ resonance. The energy transfer is manifested by a heteroclinic connection, which connects the purely symmetric sloshing modes to the purely antisymmetric sloshing modes. In [32, 35] the dynamic coupling problem is studied for a suspended rectangular container from a single rigid pivoting rod and with interior partially filled with an inviscid fluid.

The interest in this paper is to extend the Hamiltonian formulation and symplectic numerical integration of the dynamic coupling problem reported in [3] in two directions: to consider variable cross-section and bottom topography of the vessel in the shallow-water model, and to include nonlinearity in the vessel motion.

The shallow-water equations over variable cross-section and bottom topography form a set of nonlinear hyperbolic conservation laws with geometric source terms due to the arbitrary crosssection constraining the flow. For a vessel with a symmetric rectangular cross-section with regard to a vertical plane passing through its longitudinal horizontal axis, the equations take the form

$$
\begin{aligned}
(h \sigma)_{t}+(h u \sigma)_{x} & =0, \\
(h u \sigma)_{t}+\left(h u^{2} \sigma+\frac{1}{2} g h^{2} \sigma\right)_{x} & =\frac{1}{2} g h^{2} \sigma_{x}-g h \sigma \beta_{x},
\end{aligned}
$$

where $g$ is the gravitational constant, $h(x, t)$ is the fluid depth, $u(x, t)$ is the vertically averaged horizontal fluid velocity, $\beta(x)$ is the bottom topography, and $\sigma(x)$ is the breadth function such that the vessel walls are defined by the equations $y= \pm \frac{1}{2} \sigma(x)$. In [38] extensions of $Q$-schemes of van Leer and Roe are proposed for the numerical solution of the hyperbolic 


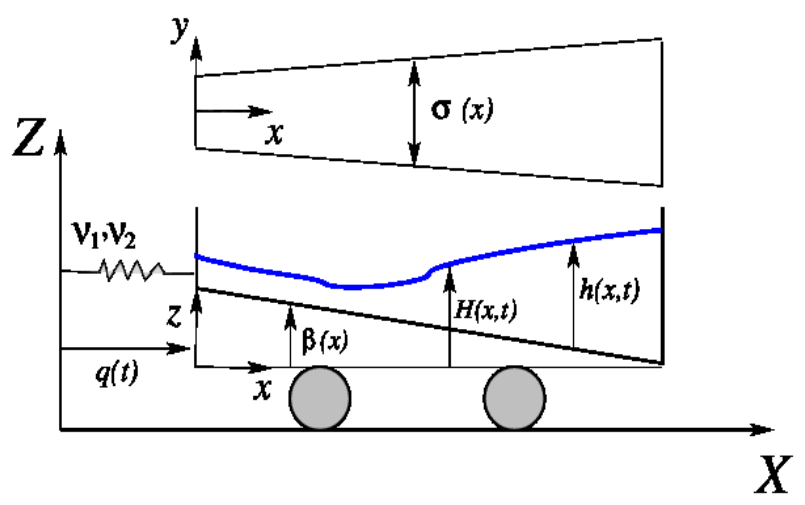

Figure 1: Schematic of shallow-water sloshing inside a vessel with variable bottom topography $\beta(x)$ and variable cross-section $\sigma(x)$ which is attached to a nonlinear spring.

system (1.1). In $[16,15,19]$ approximate Riemann solutions of the shallow-water equations over constant and variable cross-section based on the Roe linearization method are presented. In [5] a class of augmented aproximate Riemann solvers and Roe-type solvers are proposed to solve the shallow-water equations (1.1) in a moving vessel with wetting and drying. But a numerical scheme for the problem of dynamic coupling between shallow-water equations over variable cross-section and bottom topography and the nonlinear horizontal vessel motion, which respects the partition of energy between the fluid and vessel, is not developed yet. The purpose of the research reported here in this paper is to present such numerical method.

The momentum equation in the shallow-water system (1.1) can be modified to bring in the horizontal acceleration of the vessel $\ddot{q}$, relative to the spatial coordinate system $(X, Y, Z)$, into the formulation. A schematic of the configuration of interest is shown in Figure 1. Then the shallow-water equations relative to the coordinate system $(x, y, z)$ attached to the moving vessel are the mass conservation equation in (1.1) and the momentum equation

$$
(h u \sigma)_{t}+\left(h u^{2} \sigma+\frac{1}{2} g h^{2} \sigma\right)_{x}=\frac{1}{2} g h^{2} \sigma_{x}-g h \sigma \beta_{x}-\ddot{q} h \sigma .
$$

The momentum equation (1.2) can be written in the following form which is suitable for transformation to the Lagrangian particle path setting

$$
u_{t}+u u_{x}+g h_{x}=-g \beta_{x}-\ddot{q} .
$$

To summarize we will be working on the shallow-water system

$$
\begin{aligned}
& (h \sigma)_{t}+(h u \sigma)_{x}=0, \\
& u_{t}+u u_{x}+g h_{x}=-g \beta_{x}-\ddot{q},
\end{aligned}
$$

on the interval $0 \leq x \leq L$ with the boundary conditions

$$
u(0, t)=u(L, t)=0, \text { for all } t
$$

The nonlinear vessel equation with interior shallow-water sloshing can be derived with Newton's second law for the coupled system, or it can be derived by using a variational principle 
[2]. The vessel equation is

$$
\frac{d}{d t}\left(\int_{0}^{L} \rho h u \sigma d x+\left(m_{v}+m_{f}\right) \dot{q}\right)+\nu_{1} q-\nu_{2} q^{3}=0,
$$

where $q(t)$ is the horizontal position of the vessel relative to the fixed coordinate system $(X, Y, Z), \nu_{1}>0$ and $\nu_{2}$ are spring constants, $\rho$ is the fluid density, $m_{v}$ is the mass of the vessel, and $m_{f}$ is the mass of the fluid

$$
m_{f}=\int_{0}^{L} \int_{\beta(x)}^{H(x, t)} \rho \sigma(x) d z d x
$$

where $H(x, t)=\beta(x)+h(x, t)$ represents the wave elevation with respect to $z=0$. The equation of the free surface is $z=H(x, t)$, and the equation of the bottom topography is $z=\beta(x)$. When $q(t)$ is given, equation (1.6) is not used and the shallow-water equations (1.4) are forced by the time-dependent function $\ddot{q}(t)$. This problem is studied in [5] and has been extensively studied for the pure shallow-water equations without geometric source terms (e.g. $[39,7,27,28,22,9])$, and it has been shown to lead to very complicated motion. One measure of the difference with the coupled case is the role of energy. In the case of forced motion, the energy is not conserved

$$
\frac{d E}{d t}=-\ddot{q} \int_{0}^{L} \rho h u \sigma d x
$$

where

$$
E(t)=\int_{0}^{L}\left(\frac{1}{2} g\left(H^{2}-\beta^{2}\right)+\frac{1}{2} h u^{2}\right) \rho \sigma d x
$$

On the other hand the energy of the coupled problem is conserved: $\frac{d E_{c}}{d t}=0$ where

$$
E_{c}=\int_{0}^{L}\left(\frac{1}{2} g\left(H^{2}-\beta^{2}\right)+\frac{1}{2} h(u+\dot{q})^{2}\right) \rho \sigma d x+\frac{1}{2} m_{v} \dot{q}^{2}+\frac{1}{2} \nu_{1} q^{2}-\frac{1}{4} \nu_{2} q^{4} .
$$

The vessels's potential energy is expressed in the last two terms together. The third-to-last term in equation (1.7) is the vessel's kinetic energy.

The paper starts with the Lagrangian particle path formulation of the coupled shallow-water and vessel equations (1.4) and (1.6) and also derivation of a Lagrangian variational principle for the coupled system in $\S 2$. The Hamiltonian formulation for the coupled variational principle is given in $\S 3$. In $\S 4$ the Hamiltonian functional is discretized and the semi-discretization of the coupled Hamiltonian equations is given. The full symplectic time discretization using the Störmer-Verlet algorithm is given in $§ 5$. Some numerical results are presented and discussed in $\S 6$. Finally the paper ends with some concluding remarks in $\$ 7$.

\section{Lagrangian particle path formulation of the fluid and the vessel motion}

In computational fluid dynamics, the Eulerian fluid representation is preferred over the Lagrangian representation since mesh distortion can cause problems. However in one space di- 
mension mesh distortion is not a problem. Indeed, the Lagrangian particle path (LPP) representation of the equations takes an elegant and simple form. Let $x(a, t)$ be the position of a fluid particle. To derive the LPP form of the equations consider the mapping

$$
(t, a) \mapsto(t, x(a, t)), \quad \text { with } \quad 0 \leq a \leq L, \quad t \geq 0 .
$$

Assuming non-degeneracy $\left(x_{a} \neq 0\right)$ the derivatives in (1.4) are mapped to

$$
\frac{\partial}{\partial t} \mapsto \frac{\partial}{\partial t}-x_{t} x_{a}^{-1} \frac{\partial}{\partial a} \quad \text { and } \quad \frac{\partial}{\partial x} \mapsto x_{a}^{-1} \frac{\partial}{\partial a} .
$$

Substitute into (1.4), a pair of equations is being handled,

$$
\begin{aligned}
& (h \sigma)_{t}-x_{t} x_{a}^{-1}(h \sigma)_{a}+x_{a}^{-1}(h u \sigma)_{a}=0 \\
& u_{t}-x_{t} x_{a}^{-1} u_{a}+u x_{a}^{-1} u_{a}+g x_{a}^{-1} h_{a}=-g \beta_{x}(x(a, t))-\ddot{q} .
\end{aligned}
$$

Setting $u=x_{t}$ and multiplying the first equation by $x_{a}$ simplifies these equations to

$$
\begin{aligned}
x_{a}(h \sigma)_{t}+h \sigma u_{a} & =0 \\
x_{t t}+g x_{a}^{-1} h_{a} & =-g \beta_{x}(x(a, t))-\ddot{q} .
\end{aligned}
$$

The first equation gives that $h \sigma x_{a}$ is independent of time and so

$$
h(a, t) \sigma(x(a, t)) x_{a}=\left.h \sigma x_{a}\right|_{t=0}, \quad \text { hence define } \quad \chi(a):=\left.h \sigma x_{a}\right|_{t=0} .
$$

The second equation of (2.10) then reduces to

$$
\frac{\partial^{2} x}{\partial t^{2}}+\frac{g}{x_{a}} \frac{\partial}{\partial a}\left(\frac{\chi}{x_{a} \sigma(x(a, t))}\right)=-g \beta_{x}(x(a, t))-\ddot{q},
$$

or

$$
x_{t t}+\frac{g \chi_{a}}{x_{a}^{2} \sigma(x(a, t))}-\frac{g x_{a a} \chi}{x_{a}^{3} \sigma(x(a, t))}-\frac{g \sigma_{x}(x(a, t)) \chi}{x_{a} \sigma^{2}(x(a, t))}=-g \beta_{x}(x(a, t))-\ddot{q} .
$$

The equation for the vessel motion also needs to be transformed to the LPP setting. The vessel equation (1.6) in the LPP setting reads

$$
\frac{d^{2}}{d t^{2}}\left(\int_{0}^{L} x(a, t) \rho \chi(a) d a+\left(m_{v}+m_{f}\right) q(t)\right)+\nu_{1} q(t)-\nu_{2} q^{3}(t)=0 .
$$

\subsection{Lagrangian variational principle for the coupled problem}

The coupled LPP equations (2.12) and (2.13) for $x(a, t)$ and $q(t)$ can be completely determined by the Euler-Lagrange equation of the Lagrangian functional

$$
\begin{aligned}
\mathcal{L}(x, q)= & \int_{t_{1}}^{t_{2}} \int_{0}^{L}\left(\frac{1}{2}(\dot{x}+\dot{q})^{2}-\frac{1}{2} g \frac{\chi(a)}{x_{a} \sigma(x(a, t))}-g \beta(x(a, t))\right) \rho \chi(a) d a d t \\
& +\int_{t_{1}}^{t_{2}}\left(\frac{1}{2} m_{v} \dot{q}^{2}-\frac{1}{2} \nu_{1} q^{2}+\frac{1}{4} \nu_{2} q^{4}\right) \mathrm{d} t .
\end{aligned}
$$


The advantage of the variational principle is that it is much easier to design a numerical scheme with good energy conservation properties. The Euler-Lagrange equations also have a Hamiltonian formulation. This structure suggests that a symplectic integrator can be used. In this paper a variant of the Störmer-Verlet algorithm is used. This algorithm has excellent energy and momentum conservation properties [23, 18], and it is explicit.

Taking the first variation of the Lagrangian action (2.14) with respect to $q$ gives

$$
\begin{aligned}
\left.\frac{d}{d s}\right|_{s=0} \mathcal{L}(x, q+s \widehat{q}) & =\int_{t_{1}}^{t_{2}} \widehat{q}_{t} \int_{0}^{L}\left(x_{t}+q_{t}\right) \rho \chi(a) d a d t+\int_{t_{1}}^{t_{2}}\left(m_{v} \widehat{q}_{t} q_{t}-\nu_{1} \widehat{q} q+\nu_{2} \widehat{q} q^{3}\right) d t \\
& =\int_{t_{1}}^{t_{2}} \widehat{q}_{t}\left[\int_{0}^{L} x_{t} \rho \chi(a) d a+\left(m_{f}+m_{v}\right) q_{t}\right] d t-\int_{t_{1}}^{t_{2}}\left(\nu_{1} q-\nu_{2} q^{3}\right) \widehat{q} d t .
\end{aligned}
$$

Integrating by parts and using fixed endpoint conditions on the variations $\widehat{q}$ gives the equation for $q$ in (2.13).

Now take the first variation of the action integral (2.14) with respect to $x$,

$$
\begin{aligned}
& \left.\frac{d}{d s}\right|_{s=0} \mathcal{L}(x+s \widehat{x}, q)= \\
& \int_{t_{1}}^{t_{2}} \int_{0}^{L}\left(\left(x_{t}+q_{t}\right) \widehat{x}_{t}+\frac{g}{2} \frac{\chi(a)}{x_{a}^{2} \sigma(x(a, t))} \widehat{x}_{a}+\frac{g}{2} \frac{\sigma_{x}(x(a, t)) \chi(a) \widehat{x}}{x_{a} \sigma^{2}(x(a, t))}\right. \\
& \left.-g \beta_{x}(x(a, t)) \widehat{x}\right) \rho \chi(a) d a d t
\end{aligned}
$$

where integration by parts has been used to obtain the second equation, with boundary terms neglected. Setting this expression to zero recovers the LPP equation for $x(a, t)$ in (2.12).

\section{Hamiltonian formulation}

The coupled system (2.12) and (2.13) can also be expressed in Hamiltonian form with canonical variables $(q, x, p, w)$. The momentum variables are

$$
\begin{aligned}
w(a, t) & =x_{t}(a, t)+q_{t}(t) \\
p(t) & =m_{v} q_{t}(t)+\int_{0}^{L} w(a, t) \rho \chi(a) d a .
\end{aligned}
$$

It is a mixed system in the sense that $x(a, t), w(a, t)$ depend on $a$ as well as $t$ but $q(t), p(t)$ are dependent on $t$ only. The Hamiltonian formulation is derived by taking the Legendre transform of $\mathcal{L}$. The Hamiltonian functional is

$$
\begin{aligned}
\mathcal{H}(x, q, w, p)= & \int_{0}^{L} \frac{1}{2} w^{2} \rho \chi(a) d a-\frac{1}{m_{v}} p \int_{0}^{L} w \rho \chi(a) d a+\frac{1}{2} \frac{1}{m_{v}} p^{2}+\frac{1}{2} \frac{1}{m_{v}}\left(\int_{0}^{L} w \rho \chi(a) d a\right)^{2} \\
& +\frac{1}{2} \nu_{1} q^{2}-\frac{1}{4} \nu_{2} q^{4}+\int_{0}^{L}\left(\frac{1}{2} \frac{\chi(a)}{x_{a} \sigma(x(a, t))}+\beta(x(a, t))\right) \rho g \chi(a) d a .
\end{aligned}
$$


The governing equations in Hamiltonian form are

$$
\begin{aligned}
\dot{w} & =-\frac{\delta \mathcal{H}}{\delta x}=-\frac{g \chi_{a}}{x_{a}^{2} \sigma(x(a, t))}+\frac{g x_{a a} \chi}{x_{a}^{3} \sigma(x(a, t))}+\frac{g \sigma_{x}(x(a, t)) \chi}{x_{a} \sigma^{2}(x(a, t))}-g \beta_{x}(x(a, t)) \\
\dot{p} & =-\frac{\delta \mathcal{H}}{\delta q}=-\nu_{1} q+\nu_{2} q^{3} \\
\dot{x} & =\frac{\delta \mathcal{H}}{\delta w}=w-\frac{1}{m_{v}} p+\frac{1}{m_{v}} \int_{0}^{L} w \rho \chi(a) d a \\
\dot{q} & =\frac{\delta \mathcal{H}}{\delta p}=\frac{1}{m_{v}} p-\frac{1}{m_{v}} \int_{0}^{L} w \rho \chi(a) d a .
\end{aligned}
$$

The gradient of $\mathcal{H}$ is taken with respect to a weighted inner product. Let $Z=(x, q, w, p)$. Then the inner product is defined by

$$
\left\langle\left\langle Z_{1}, Z_{2}\right\rangle\right\rangle:=\int_{0}^{L}\left(x_{1} x_{2}+w_{1} w_{2}\right) \rho \chi d a+q_{1} q_{2}+p_{1} p_{2} .
$$

Hence the gradient of $\mathcal{H}$ is defined by

$$
\left.\frac{d}{d s} \mathcal{H}(Z+s \delta Z)\right|_{s=0}=\langle\langle\nabla \mathcal{H}, \delta Z\rangle\rangle=\int_{0}^{L}\left(\frac{\delta \mathcal{H}}{\delta x} \delta x+\frac{\delta \mathcal{H}}{\delta w} \delta w\right) \rho \chi d a+\frac{\delta \mathcal{H}}{\delta q} \delta q+\frac{\delta \mathcal{H}}{\delta p} \delta p
$$

Associated with the system (3.17) are the boundary conditions

$$
x(0, t)=0 \quad \text { and } \quad x(L, t)=L, \quad \text { for all } t,
$$

and

$$
w(0, t)=w(L, t)=\frac{1}{m_{v}} p-\frac{1}{m_{v}} \int_{0}^{L} w(a, t) \rho \chi(a) d a, \quad \text { for all } t .
$$

The symplectic form associated with this Hamiltonian formulation is

$$
\Omega=\int_{0}^{L} \mathrm{~d} w \wedge d x \rho \chi d a+d p \wedge d q .
$$

The Hamiltonian functional (3.16) is the total energy, and it is conserved along solutions.

\section{Numerical algorithm - the semi-discretization}

Discretize the reference space by letting

$$
a_{i}=(i-1) \Delta a, \quad i=1, \ldots, N+1, \quad \text { with } \quad \Delta a=\frac{L}{N},
$$

and let $x_{i}(t):=x\left(a_{i}, t\right)$ and $w_{i}(t):=w\left(a_{i}, t\right)$. After choosing a discretization for the derivatives and integrals with respect to $a$, the governing equations can be written as a large set of ordinary differential equations. There are only two tricky points: how to discretize the righthand side of the first equation in (3.17), and secondly how to discretize the integral term in the third and fourth equations. 
For the first difficulty, a variational discretization is used [25]: discretize the Hamiltonian functional first, and then take a variation of the discretized Hamiltonian [25]. In this case the functional that generates the term is

$$
\mathcal{F}(x)=\int_{0}^{L}-\frac{g \chi(a)}{2 x_{a} \sigma(x(a, t))} \rho \chi(a) d a .
$$

Discretize using a finite difference formula for the derivative $x_{a}$

$$
\mathcal{F}\left(x_{1}, \ldots, x_{N+1}\right)=\sum_{i=1}^{N}-\frac{1}{2} \frac{g \chi_{i} \Delta a}{\left(x_{i+1}-x_{i}\right) \sigma_{i}} \rho \chi_{i} \Delta a
$$

where $\sigma_{i}=\sigma\left(x_{i}\right)$. Now take a variation in $\left(x_{1}, \ldots, x_{N+1}\right)$,

$$
\begin{aligned}
& \left.\frac{d}{d s}\right|_{s=0} \mathcal{F}\left(x_{1}+s \xi_{1}, \ldots, x_{N+1}+s \xi_{N+1}\right)= \\
& \sum_{i=2}^{N}\left(\frac{g}{2 \chi_{i}}\left(\frac{\chi_{i-1}^{2} \Delta a}{\left(x_{i}-x_{i-1}\right)^{2} \sigma_{i-1}}-\frac{\chi_{i}^{2} \Delta a}{\left(x_{i+1}-x_{i}\right)^{2} \sigma_{i}}\right)+\frac{g \Delta a}{2} \frac{\left(\sigma_{x}\right)_{i} \chi_{i}}{\left(x_{i+1}-x_{i}\right) \sigma_{i}^{2}}\right) \rho \xi_{i} \chi_{i} \Delta a .
\end{aligned}
$$

This argument suggests that the natural semi-discretization of the first equation in (3.17) is then

$$
\begin{gathered}
\dot{w}=\frac{g \Delta a}{2 \chi_{i}}\left(\frac{\chi_{i-1}^{2}}{\left(x_{i}-x_{i-1}\right)^{2} \sigma\left(x_{i-1}\right)}-\frac{\chi_{i}^{2}}{\left(x_{i+1}-x_{i}\right)^{2} \sigma\left(x_{i}\right)}\right) \\
+\frac{g \Delta a}{2} \frac{\sigma_{x}\left(x_{i}\right) \chi_{i}}{\left(x_{i+1}-x_{i}\right) \sigma^{2}\left(x_{i}\right)}-g \beta_{x}\left(x_{i}\right), \quad i=2, \ldots, N
\end{gathered}
$$

For the integral of $w$ that appears in (3.17) the trapezoidal rule is used

$$
\sigma_{N}(t):=\sum_{i=2}^{N} w_{i}(t) \rho \chi_{i} \Delta a+\frac{1}{2} \rho\left(\chi_{1} w_{1}(t)+\chi_{N+1} w_{N+1}(t)\right) \Delta a .
$$

The choice of quadrature formula for $\sigma_{N}$ will affect the symplecticity of the semi-discretization. Simpson's rule results in a non-symplectic semi-discretization.

The full semi-discretization is then

$$
\begin{aligned}
\dot{w}_{i}= & \frac{g \Delta a}{2 \chi_{i}}\left(\frac{\chi_{i-1}^{2}}{\left(x_{i}-x_{i-1}\right)^{2} \sigma\left(x_{i-1}\right)}-\frac{\chi_{i}^{2}}{\left(x_{i+1}-x_{i}\right)^{2} \sigma\left(x_{i}\right)}\right) \\
& +\frac{g \Delta a}{2} \frac{\sigma_{x}\left(x_{i}\right) \chi_{i}}{\left(x_{i+1}-x_{i}\right) \sigma^{2}\left(x_{i}\right)}-g \beta_{x}\left(x_{i}\right), \quad i=2, \ldots, N, \\
\dot{p}= & -\nu_{1} q+\nu_{2} q^{3}, \\
\dot{x}_{i}= & w_{i}-\frac{1}{m_{v}} p+\frac{1}{m_{v}} \sigma_{N}, \quad i=2, \ldots, N, \\
\dot{q}= & \frac{1}{m_{v}} p-\frac{1}{m_{v}} \sigma_{N},
\end{aligned}
$$

with boundary conditions

$$
x_{1}(t)=0, \quad x_{N+1}(t)=L, \quad \text { and } \quad w_{1}(t)=w_{N+1}(t)=\frac{1}{m_{v}}\left(p(t)-\sigma_{N}(t)\right) .
$$


The semi-discretization is symplectic with symplectic form

$$
\Omega_{N}=\sum_{i=2}^{N} d w_{i} \wedge d x_{i} \rho \chi_{i} \Delta a+d p \wedge d q .
$$

The semi-discretization is Hamiltonian and the Hamiltonian functional is a discretization of the energy,

$$
\begin{aligned}
\mathcal{H}_{N}= & \frac{1}{2} \rho \sum_{i=2}^{N} \chi_{i} w_{i}^{2} \Delta a+\frac{1}{4} \rho\left(\chi_{1} w_{1}^{2}+\chi_{N+1} w_{N+1}^{2}\right) \Delta a+\sum_{i=1}^{N+1} \rho g \chi_{i} \beta\left(x_{i}\right) \Delta a \\
& +\frac{1}{2} \rho g \sum_{i=1}^{N} \frac{\chi_{i}^{2} \Delta a^{2}}{\left(x_{i+1}-x_{i}\right) \sigma\left(x_{i}\right)}+\frac{1}{2} \frac{1}{m_{v}}\left(p-\sigma_{N}\right)^{2}+\frac{1}{2} \nu_{1} q^{2}-\frac{1}{4} \nu_{2} q^{4} .
\end{aligned}
$$

This energy is conserved along orbits of the semi-discretization. This latter property can be verified by introducing an appropriate inner product for the semi-discretization and using the fact that $\mathcal{H}_{N}$ is the Hamiltonian function that generates the equations (4.23).

\section{Time discretization using the Störmer-Verlet algorithm}

The semi-discretization (4.23) is of the form

$$
\dot{\mathbf{p}}=g(\mathbf{q}) \quad \text { and } \quad \dot{\mathbf{q}}=f(\mathbf{p}),
$$

where

$$
\mathbf{p}=\left(p, w_{2}, \ldots, w_{N}\right) \quad \text { and } \quad \mathbf{q}=\left(q, x_{2}, \ldots, x_{N}\right) .
$$

The precise form of $f(\cdot)$ and $g(\cdot)$ can be deduced from (4.23) but will not be needed. The important point here is the fact that the right hand side of the $\dot{\mathbf{p}}$ equation depends only on $\mathbf{q}$ and the right hand side of the $\dot{\mathbf{q}}$ equation depends only on $\mathbf{p}$. There are appealing numerical methods for equations of this form: split-step methods, or partitioned Runge-Kutta methods (e.g. Section 2.5 of [23]). One of the simplest of this class of methods is the discretization

$$
\begin{aligned}
\mathbf{p}^{n+\frac{1}{2}} & =\mathbf{p}^{n}+\frac{1}{2} \Delta \operatorname{tg}\left(\mathbf{q}^{n}\right) \\
\mathbf{q}^{n+1} & =\mathbf{q}^{n}+\Delta t f\left(\mathbf{p}^{n+\frac{1}{2}}\right) \\
\mathbf{p}^{n+1} & =\mathbf{p}^{n+\frac{1}{2}}+\frac{1}{2} \Delta t g\left(\mathbf{q}^{n+1}\right) .
\end{aligned}
$$

This scheme is explicit, and has second-order accuracy in time. If in addition the equations are Hamiltonian as in (4.23) then this scheme is called the Störmer-Verlet method and it has additional properties [23, 18]. It is symplectic, and has excellent energy conservation over long time intervals.

Applying the Störmer-Verlet algorithm to the semi-discretization (4.23) then gives the following 
equations for each time step $n \mapsto n+1$

$$
\begin{aligned}
& p^{n+\frac{1}{2}}=p^{n}-\frac{\Delta t}{2} \nu_{1} q^{n}+\frac{\Delta t}{2} \nu_{2}\left(q^{n}\right)^{3}, \\
& w_{i}^{n+\frac{1}{2}}=w_{i}^{n}+\frac{g \Delta t \Delta a}{4 \chi_{i}}\left(\frac{\chi_{i-1}^{2}}{\left(x_{i}^{n}-x_{i-1}^{n}\right)^{2} \sigma\left(x_{i-1}^{n}\right)}-\frac{\chi_{i}^{2}}{\left(x_{i+1}^{n}-x_{i}^{n}\right)^{2} \sigma\left(x_{i}^{n}\right)}\right) \\
& +\frac{g \Delta t \Delta a}{4} \frac{\sigma_{x}\left(x_{i}^{n}\right) \chi_{i}}{\left(x_{i+1}^{n}-x_{i}^{n}\right) \sigma^{2}\left(x_{i}^{n}\right)}-\frac{g \Delta t}{2} \beta_{x}\left(x_{i}^{n}\right), \quad i=2, \ldots, N, \\
& x_{i}^{n+1}=x_{i}^{n}+\Delta t w_{i}^{n+\frac{1}{2}}-\frac{\Delta t}{m_{v}} p^{n+\frac{1}{2}}+\frac{\Delta t}{m_{v}} \sigma_{N}^{n+\frac{1}{2}}, \quad i=2, \ldots, N, \\
& q^{n+1}=q^{n}+\frac{\Delta t}{m_{v}} p^{n+\frac{1}{2}}-\frac{\Delta t}{m_{v}} \sigma_{N}^{n+\frac{1}{2}} \\
& p^{n+1}=p^{n+\frac{1}{2}}-\frac{\Delta t}{2} \nu_{1} q^{n+1}+\frac{\Delta t}{2} \nu_{2}\left(q^{n+1}\right)^{3}, \\
& w_{i}^{n+1}=w_{i}^{n+\frac{1}{2}}+\frac{g \Delta t \Delta a}{4 \chi_{i}}\left(\frac{\chi_{i-1}^{2}}{\left(x_{i}^{n+1}-x_{i-1}^{n+1}\right)^{2} \sigma\left(x_{i-1}^{n+1}\right)}-\frac{\chi_{i}^{2}}{\left(x_{i+1}^{n+1}-x_{i}^{n+1}\right)^{2} \sigma\left(x_{i}^{n+1}\right)}\right) \\
& +\frac{g \Delta t \Delta a}{4} \frac{\sigma_{x}\left(x_{i}^{n+1}\right) \chi_{i}}{\left(x_{i+1}^{n+1}-x_{i}^{n+1}\right) \sigma^{2}\left(x_{i}^{n+1}\right)}-\frac{g \Delta t}{2} \beta_{x}\left(x_{i}^{n+1}\right), \quad i=2, \ldots, N
\end{aligned}
$$

where

$$
w_{i}^{n}=w\left(a_{i}, t_{n}\right), \quad x_{i}^{n}=x\left(a_{i}, t_{n}\right), \quad p^{n}=p\left(t_{n}\right), \quad q^{n}=q\left(t_{n}\right), \quad t_{n}=n \Delta t,
$$

and the boundary conditions are

$$
x_{1}^{n+1}=0 \quad \text { and } \quad x_{N+1}^{n+1}=L,
$$

and

$$
w_{1}^{n+\frac{1}{2}}=w_{N+1}^{n+\frac{1}{2}}=\frac{1}{m_{v}}\left(p^{n+\frac{1}{2}}-\sigma_{N}^{n+\frac{1}{2}}\right) .
$$

The Störmer-Verlet discretization (5.25) is symplectic. The symplectic form of the discretization is

$$
\Omega_{N}^{n}=\sum_{i=2}^{N} \delta w_{i}^{n} \wedge \delta x_{i}^{n} \rho \chi_{i} \Delta a+\delta p^{n} \wedge \delta q^{n} .
$$

This symplectic form satisfies $\Omega_{N}^{n+1}=\Omega_{N}^{n}$ for all $n$. The proof of symplecticity is given in A.

\section{Numerical results}

In this section some numerical simulations are presented for dynamic coupling between shallowwater sloshing over variable cross-section and bottom topography, and the nonlinear horizontal vessel motion using the symplectic numerical scheme (5.25). In all cases the numerical scheme is programmed in MATLAB on a Windows 7, 64 - bit Operating System with Processor of Intel(R) Core(TM) i7-3940XM CPU @ 3.20 GHz with Memory of 32 GB. 
The purpose of the first simulation is to show that the initial potential energy of the fluid causes the vessel at rest to undergo nonlinear motion, and also to show how excellent the symplectic scheme (5.25) respects the partition of energy between the fluid and vessel. For this simulation take $\rho=1000 \mathrm{~kg} / \mathrm{m}^{3}, \nu_{1}=40 \mathrm{~kg} / \mathrm{s}^{2}, \nu_{2}=30 \mathrm{~kg} / \mathrm{m}^{2} \mathrm{~s}^{2}$, and set the initial displacement and velocity of the vessel at $q(0)=0$ and $\dot{q}(0)=0$, respectively. The bottom topography is

$$
\beta(x)=0.08-0.06 x, \quad 0 \leq x \leq 1,
$$

and the vessel cross-section is taken to be

$$
\sigma(x)=0.5+0.22 x, \quad 0 \leq x \leq 1
$$

The fluid is taken to be quiescent with the initial wave profile

$$
h(a, 0)=0.02+0.1 x, \quad 0 \leq x \leq 1 .
$$

The other initial conditions and input parameters are

$$
\begin{aligned}
x(a, 0) & =a, \quad w(a, 0)=0, \quad p(0)=0, \quad m_{f}=44.5334 \mathrm{~kg}, \quad m_{v}=0.05 m_{f}=2.2266 \mathrm{~kg}, \\
\Delta a & =0.005 \mathrm{~m}, \quad \Delta t=10^{-4} \mathrm{~s} .
\end{aligned}
$$

The first panel in Figure 2 shows the computed nonlinear horizontal motion of the vessel versus time. Noting that the initial vessel displacement and velocity are zero, the initial potential energy of the fluid drives the coupled dynamics. The fluid energy has been transferred to the vessel and causes the vessel to undergo nonlinear motion governed by equation (2.13). The energy error, $\mathcal{H}_{N}(t)-\mathcal{H}_{N}(0)$, for $10^{6}$ time steps is shown in the second panel in Figure 2. This panel shows that the proposed symplectic integrator conserves the discrete energy of the coupled system (4.24) over long times. The energy error is to the order of $10^{-5}$ and it is bounded over long times.

The first panel in Figure 3 shows the energy of the vessel

$$
E_{v}=\frac{1}{2} \frac{1}{m_{v}}\left(p-\sigma_{N}\right)^{2}+\frac{1}{2} \nu_{1} q^{2}-\frac{1}{4} \nu_{2} q^{4},
$$

versus time, and the second panel in this figure shows the energy of the fluid

$E_{f}=\frac{1}{2} \rho \sum_{i=2}^{N} \chi_{i} w_{i}^{2} \Delta a+\frac{1}{4} \rho\left(\chi_{1} w_{1}^{2}+\chi_{N+1} w_{N+1}^{2}\right) \Delta a+\sum_{i=1}^{N+1} \rho g \chi_{i} \beta\left(x_{i}\right) \Delta a+\frac{1}{2} \rho g \sum_{i=1}^{N} \frac{\chi_{i}^{2} \Delta a^{2}}{\left(x_{i+1}-x_{i}\right) \sigma\left(x_{i}\right)}$,

versus time. This figure shows that the numerical scheme (5.25) respects the partition of energy between the fluid and vessel motion over long times.

The Eulerian wave profile, $h(x, t)$, is determined by plotting $h(a, t)$ versus $x(a, t)$ for fixed $t$, generating a curve parameterized by $a$. Figure 4 shows snapshots of the Eulerian free surface wave profile, $H(a, t)=h(x(a, t), t)+\beta(x(a, t))$, up to $100 \mathrm{~s}$. The slope of the Eulerian wave height is related to the slope of the wave height in the Lagrangian setting by

$$
\frac{\partial h}{\partial x}=\frac{1}{x_{a}} \frac{\partial h}{\partial a} \quad \text { where } \quad h(a, t)=\frac{\chi(a)}{x_{a} \sigma(x(a, t))} .
$$



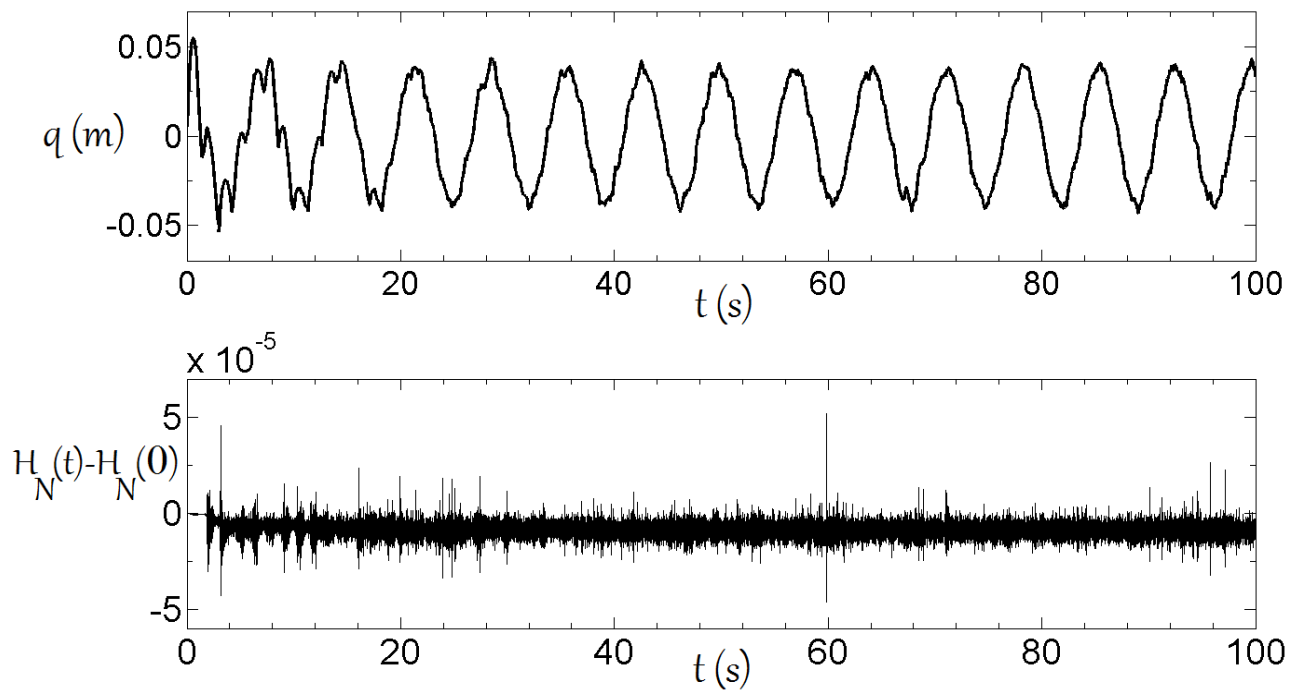

Figure 2: The fluid motion causes the vessel at rest, $q(0)=\dot{q}(0)=0$, to undergo nonlinear motion. First panel: computed nonlinear vessel motion for $100 \mathrm{~s}$. Second panel: energy error, $\mathcal{H}_{N}(t)-\mathcal{H}_{N}(0)$, for $100 \mathrm{~s}$.
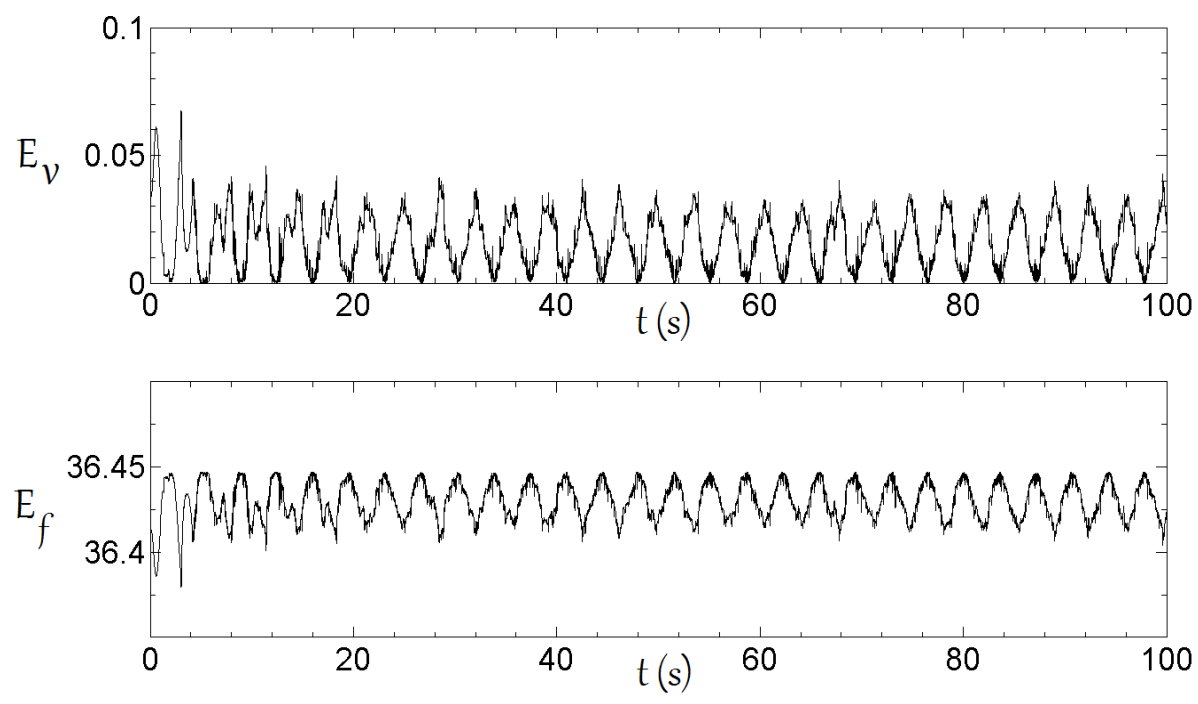

Figure 3: The partition of energy between the fluid and vessel motion. First panel: the vessel energy (6.31) versus time. Second panel: the fluid energy (6.32) versus time.

Hence, when $x_{a}$ is small, the slope is exaggerated in the Eulerian setting creating an highly nonlinear appearance. Indeed, one can create highly nonlinear solutions such as waves with cusp-like crests. For this simulation the clock time for $10^{6}$ time steps with $\Delta a=0.005 \mathrm{~m}$ is $163.60 \mathrm{~s}$.

For the next simulation take the spring constants $\nu_{1}=50 \mathrm{~kg} / \mathrm{s}^{2}, \nu_{2}=-200 \mathrm{~kg} / \mathrm{m}^{2} \mathrm{~s}^{2}$, and set the initial displacement and velocity of the vessel at $q(0)=-0.1 \mathrm{~m}$ and $\dot{q}(0)=0$, respectively. The bottom topography is (6.29). The fluid is taken to be quiescent with the initial wave profile

$$
h(a, 0)=0.1-\beta(x(a, 0)) .
$$



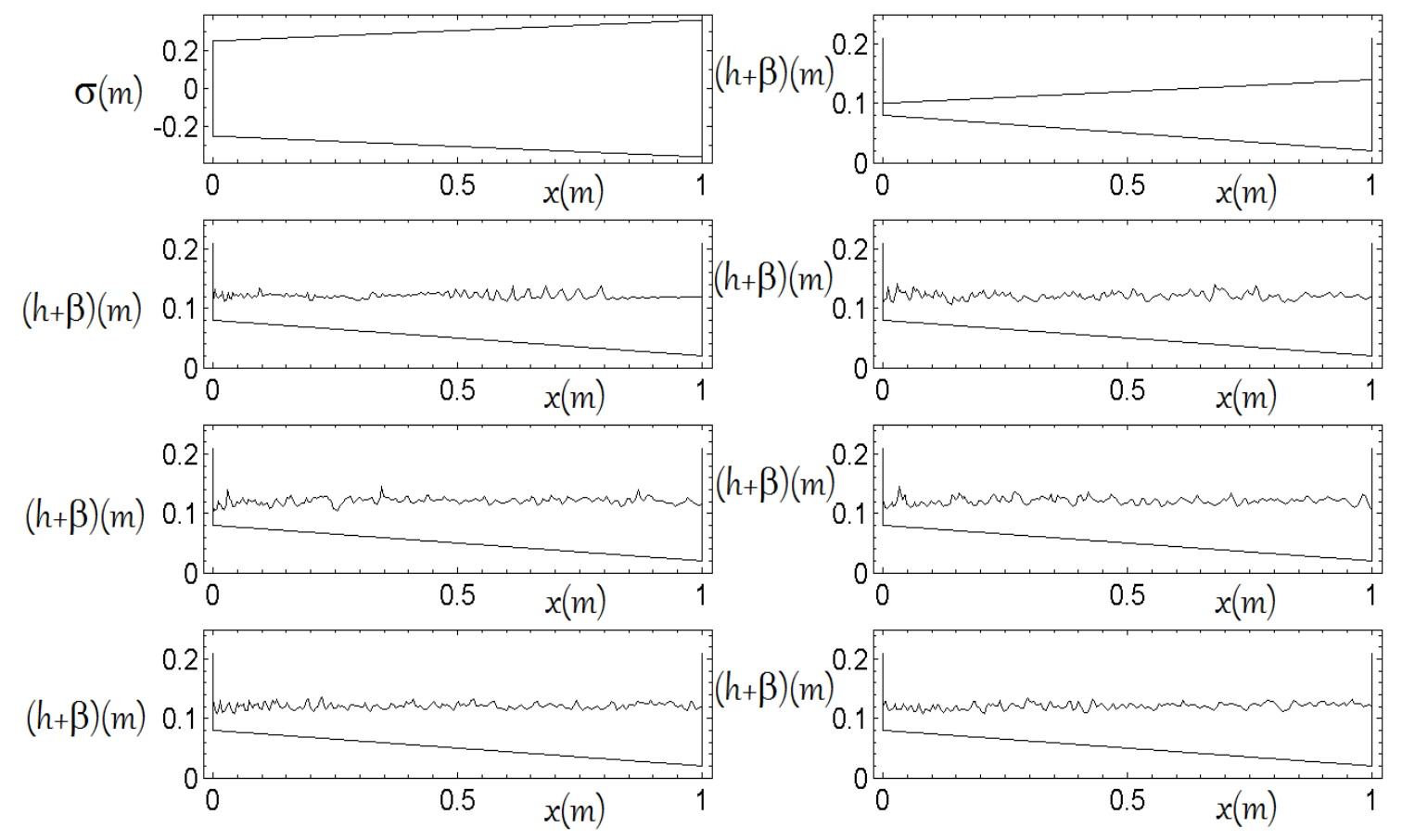

Figure 4: First row left: the vessel cross-section, horizontal axis is $x(m)$ and vertical axis is $\sigma(x)$. First row right: snapshot of the Eulerian wave profile at $t=0 \mathrm{~s}$. The horizontal and vertical axes are, $x(a, t)$ and $h(x(a, t), t)+\beta(x(a, t))$, respectively. Second row left: snapshot of the Eulerian wave profile at $t=4 \mathrm{~s}$. Second row right: snapshot of the Eulerian wave profile at $t=18 \mathrm{~s}$. Third row left: snapshot of the Eulerian wave profile at $t=36 \mathrm{~s}$. Third row right: snapshot of the Eulerian wave profile at $t=52 \mathrm{~s}$. Fourth row left: snapshot of the Eulerian wave profile at $t=84 \mathrm{~s}$. Fourth row right: snapshot of the Eulerian wave profile at $t=100 \mathrm{~s}$.

The purpose of this simulation is to show how the initial potential energy of the vessel drives the coupled dynamics, and to show that the symplectic numerical scheme (5.25) respects the partition of energy between the fluid and vessel. The vessel cross-section is taken to be

$$
\sigma(x)=0.5+0.24 x, \quad 0 \leq x \leq 1 .
$$

The other initial conditions and input parameters are

$$
\begin{aligned}
x(a, 0) & =a, \quad w(a, 0)=0, \quad p(0)=0, \quad m_{f}=32.2 \mathrm{~kg}, \quad m_{v}=0.1 m_{f}=3.22 \mathrm{~kg}, \\
\Delta a & =0.005 \mathrm{~m}, \quad \Delta t=10^{-4} \mathrm{~s}, \quad \rho=1000 \mathrm{~kg} / \mathrm{m}^{3} .
\end{aligned}
$$

The first panel in Figure 5 shows the computed horizontal motion of the vessel for $150 \mathrm{~s}$. The energy error, $\mathcal{H}_{N}(t)-\mathcal{H}_{N}(0)$, for $1.5 \times 10^{6}$ time steps is shown in the third panel in Figure 5 . This subplot shows that the proposed symplectic integrator conserves the discrete energy of the coupled system over long times. The energy error is to the order of $10^{-6}$ and it is bounded over long times.

The first panel in Figure 6 shows the energy of the vessel (6.31) versus time, and the second panel shows the energy of the fluid (6.32) versus time. This figure shows that the partition of energy between the fluid and vessel motion is maintained. Snapshots of the Eulerian free surface wave profile are shown in Figure 7 up to $150 \mathrm{~s}$. The clock time for $1.5 \times 10^{6}$ time steps with $\Delta a=0.005 \mathrm{~m}$ is $263.6395 \mathrm{~s}$. 

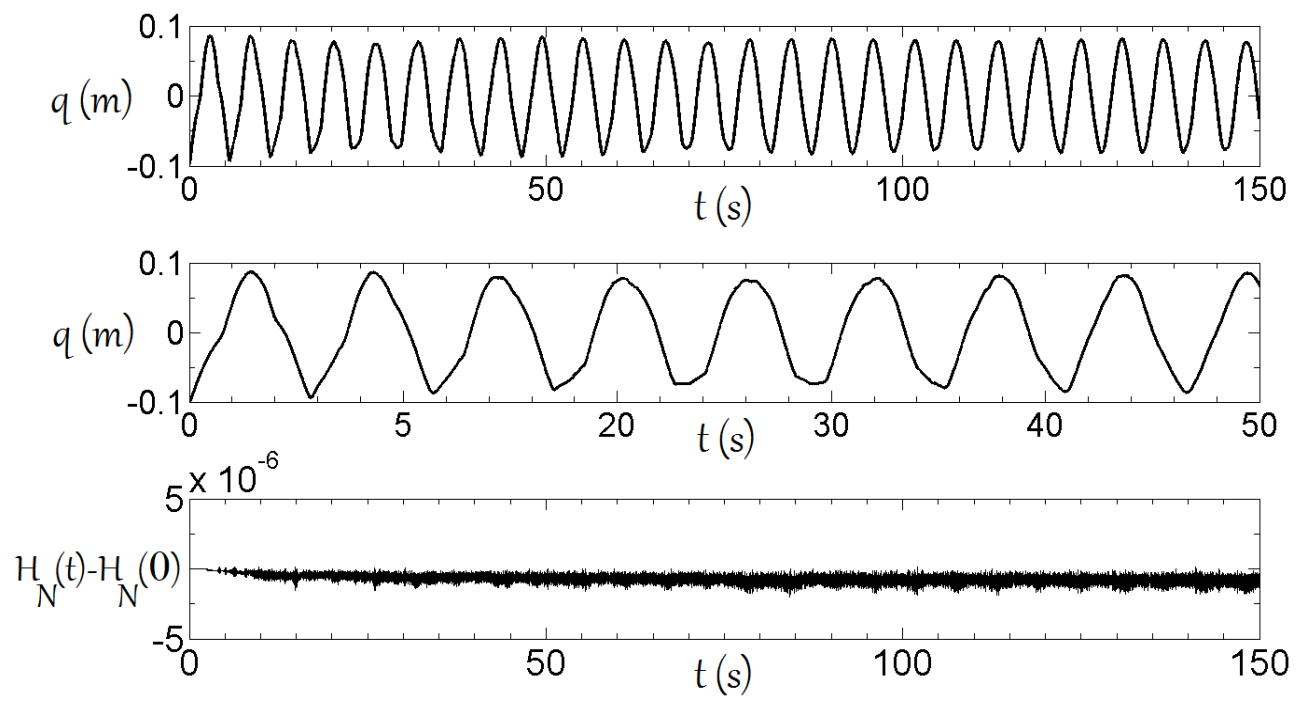

Figure 5: The initial potential energy of the vessel drives the coupled dynamics. First panel: computed nonlinear vessel motion for $150 \mathrm{~s}$. Second panel: vessel position for first $50 \mathrm{~s}$. Third panel: energy error, $\mathcal{H}_{N}(t)-\mathcal{H}_{N}(0)$, for $150 \mathrm{~s}$.
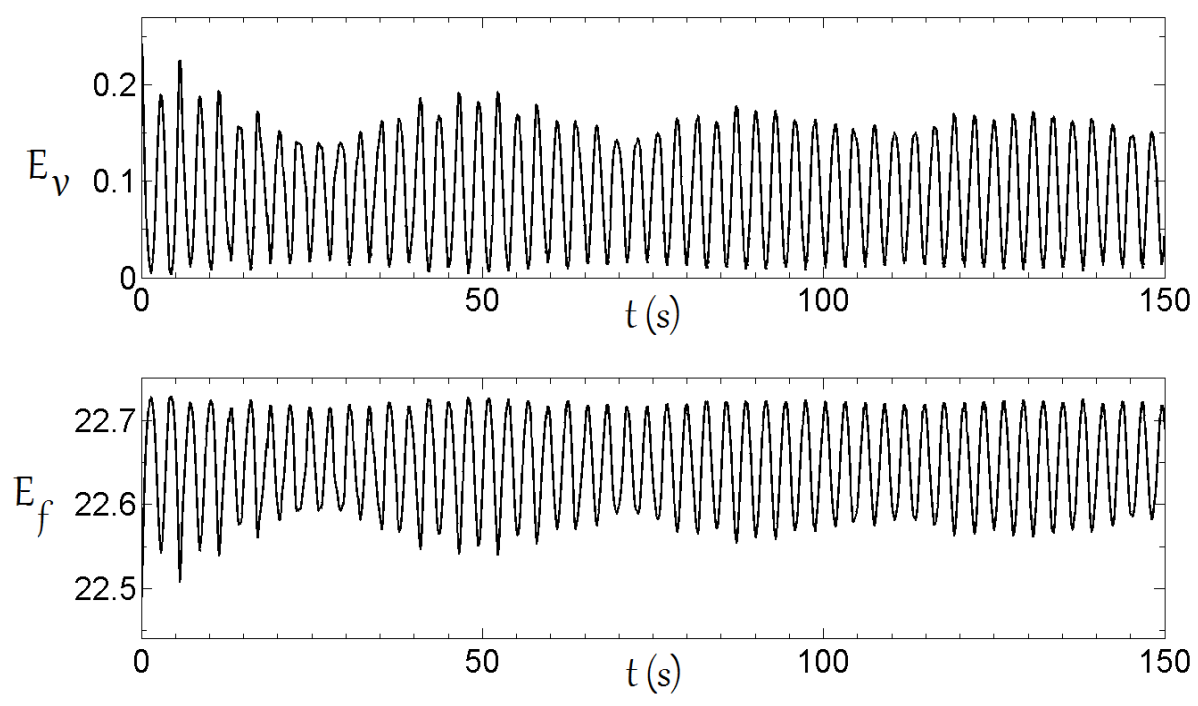

Figure 6: The partition of energy between the fluid and vessel motion. First panel: the vessel energy (6.31) versus time. Second panel: the fluid energy (6.32) versus time.

\section{Concluding remarks}

The coupled problem of nonlinear shallow-water sloshing over variable cross-section and bottom topography and nonlinear vessel dynamics in the Lagrangian particle path setting has been studied numerically. The emphasis in this paper has been development of a symplectic integrator which accurately represents the coupled dynamics and respects the energy budget. It is of interest to preserve the total energy in the numerical scheme, but also to maintain the partition of energy between the fluid and vessel. Numerical results for the coupled dynamics are presented 


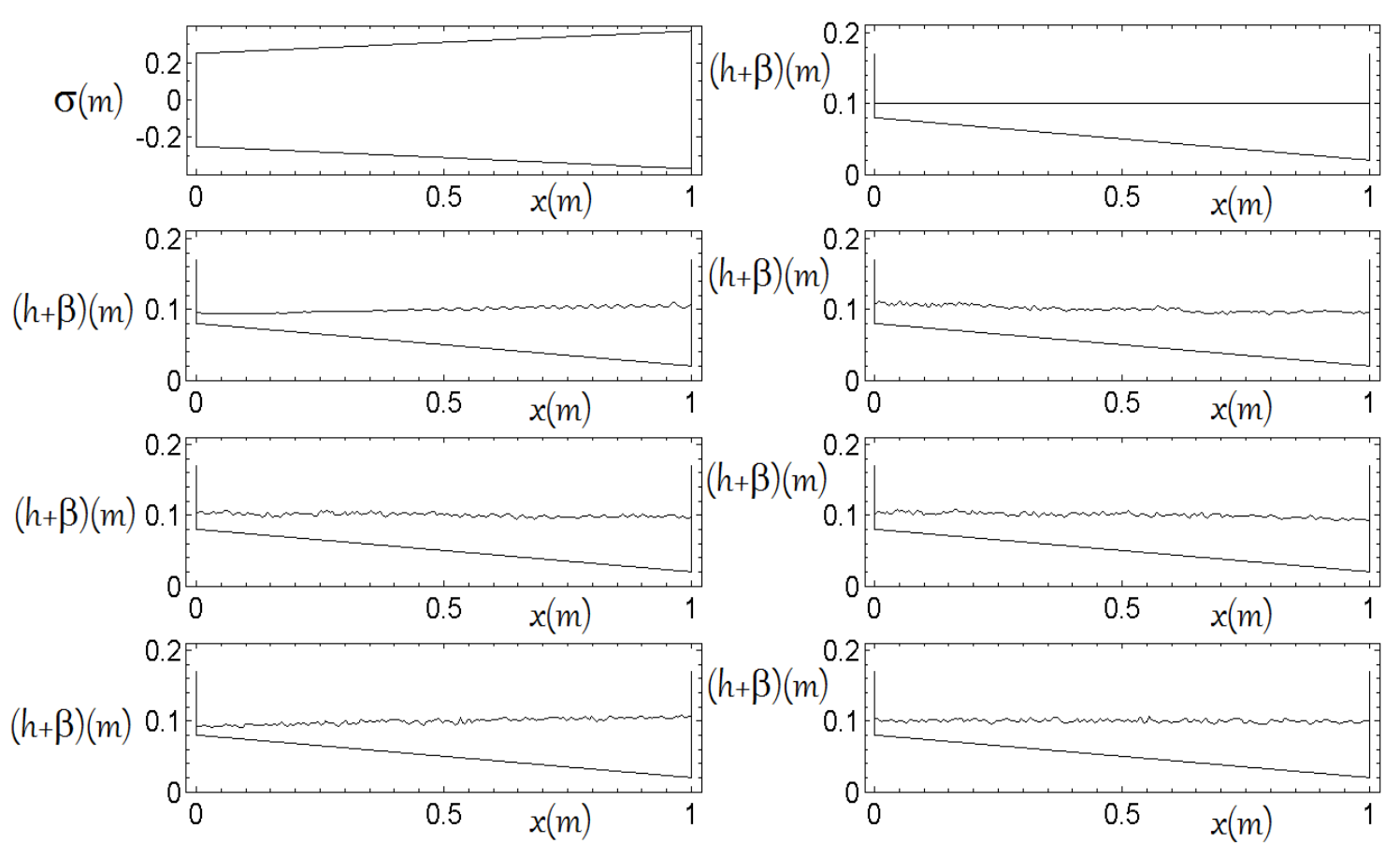

Figure 7: First row left: the vessel cross-section, horizontal axis is $x(m)$ and vertical axis is $\sigma(x)$. First row right: snapshot of the Eulerian wave profile at $t=0 \mathrm{~s}$. The horizontal and vertical axes are, $x(a, t)$ and $h(x(a, t), t)+\beta(x(a, t))$, respectively. Second row left: snapshot of the Eulerian wave profile at $t=4 \mathrm{~s}$. Second row right: snapshot of the Eulerian wave profile at $t=18 \mathrm{~s}$. Third row left: snapshot of the Eulerian wave profile at $t=36 \mathrm{~s}$. Third row right: snapshot of the Eulerian wave profile at $t=52 \mathrm{~s}$. Fourth row left: snapshot of the Eulerian wave profile at $t=84 \mathrm{~s}$. Fourth row right: snapshot of the Eulerian wave profile at $t=150 \mathrm{~s}$.

which confirm that the proposed symplectic solver conserves the total energy of the system and also respects the energy budget.

A direction of great interest is to add a prescribed quasi-periodic forcing function of the form

$$
q_{p}=\varepsilon_{1} \sin \left(\omega_{1} t\right)+\varepsilon_{2} \sin \left(\omega_{2} t\right) \quad \text { with } \quad \frac{\omega_{1}}{\omega_{2}} \text { irrational }
$$

to the vessel equation, and to extend the Hamiltonian formulation to include the forcing function. In this case the Hamiltonian functional is non-separable and an implicit symplectic integrator such as the generalized Störmer-Verlet scheme [23] or the implicit midpoint rule should be used. It is desirable to do a parametric study for possible detection of chaos in the coupled dynamics.

Another direction of great interest is to extend the presented Lagrangian particle path formulation and symplectic integration of the coupled dynamics for the problem of two-layer shallowwater sloshing over variable cross-section and bottom topography coupled to the vessel motion with a rigid-lid. The motivation is modelling of the ocean wave energy harvesters. 


\section{Acknowledgments}

The author is grateful to Thomas J. Bridges and Matthew R. Turner for useful discussions. The research reported in this paper is supported by the EPSRC under Grant number EP/K008188/1. Due to confidentiality agreements with research collaborators, supporting data can only be made available to bona fide researchers subject to a non-disclosure agreement. Details of the data and how to request access are available from the University of Surrey publications repository: researchdata@surrey.ac.uk

\section{A Proof of symplecticity of the numerical scheme (5.25)}

To test for symplecticity, the variational equations are required

$$
\begin{aligned}
\delta w_{i}^{n+\frac{1}{2}}= & \delta w_{i}^{n}+\frac{A_{i}^{n}}{\chi_{i}} \delta x_{i+1}^{n}-\frac{A_{i}^{n}}{\chi_{i}} \delta x_{i}^{n}-\frac{A_{i-1}^{n}}{\chi_{i}} \delta x_{i}^{n}+\frac{A_{i-1}^{n}}{\chi_{i}} \delta x_{i-1}^{n} \\
& +\frac{2 B_{i}^{n}}{\chi_{i}} \delta x_{i}^{n}-\frac{B_{i-1}^{n}}{\chi_{i}} \delta x_{i-1}^{n}-\frac{B_{i}^{n}}{\chi_{i}} \delta x_{i+1}^{n} \\
& +\frac{g \Delta a \Delta t}{4} \frac{\sigma_{x x}\left(x_{i}^{n}\right) \chi_{i}}{\left(x_{i+1}^{n}-x_{i}^{n}\right) \sigma^{2}\left(x_{i}^{n}\right)} \delta x_{i}^{n}-\frac{g \Delta a \Delta t}{2} \frac{\sigma_{x}^{2}\left(x_{i}^{n}\right) \chi_{i}}{\left(x_{i+1}^{n}-x_{i}^{n}\right) \sigma^{3}\left(x_{i}^{n}\right)} \delta x_{i}^{n} \\
& -\frac{g \Delta t}{2} \beta_{x x}\left(x_{i}^{n}\right) \delta x_{i}^{n}, \quad i=2, \ldots, N, \\
\delta p^{n+\frac{1}{2}}= & \delta p^{n}-\frac{1}{2} \nu_{1} \Delta t \delta q^{n}+\frac{3}{2} \nu_{2} \Delta t\left(q^{n}\right)^{2} \delta q^{n}, \\
\delta x_{i}^{n+1}= & \delta x_{i}^{n}+\Delta t \delta w_{i}^{n+\frac{1}{2}}-\frac{\Delta t}{m_{v}} \delta p^{n+\frac{1}{2}}+\frac{\Delta t}{m_{v}} \delta \sigma_{N}^{n+\frac{1}{2}}, \quad i=2, \ldots, N, \\
\delta q^{n+1}= & \delta q^{n}+\frac{\Delta t}{m_{v}} \delta p^{n+\frac{1}{2}}-\frac{\Delta t}{m_{v}} \delta \sigma_{N}^{n+\frac{1}{2}}, \\
\delta w_{i}^{n+1}= & \delta w_{i}^{n+\frac{1}{2}}+\frac{A_{i}^{n+1}}{\chi_{i}} \delta x_{i+1}^{n+1}-\frac{A_{i}^{n+1}}{\chi_{i}} \delta x_{i}^{n+1} \\
& -\frac{A_{i-1}^{n+1}}{\chi_{i}} \delta x_{i}^{n+1}+\frac{A_{i-1}^{n+1}}{\chi_{i}} \delta x_{i-1}^{n+1}+\frac{2 B_{i}^{n+1}}{\chi_{i}} \delta x_{i}^{n+1}-\frac{B_{i-1}^{n+1}}{\chi_{i}} \delta x_{i-1}^{n+1}-\frac{B_{i}^{n+1}}{\chi_{i}} \delta x_{i+1}^{n+1} \\
& +\frac{g \Delta a \Delta t}{4} \frac{\sigma_{x x}\left(x_{i}^{n+1}\right) \chi_{i}}{\left(x_{i+1}^{n+1}-x_{i}^{n+1}\right) \sigma^{2}\left(x_{i}^{n+1}\right)} \delta x_{i}^{n+1}-\frac{g \Delta a \Delta t}{2} \frac{\sigma_{x}^{2}\left(x_{i}^{n+1}\right) \chi_{i}}{\left(x_{i+1}^{n+1}-x_{i}^{n+1}\right) \sigma^{3}\left(x_{i}^{n+1}\right)} \delta x_{i}^{n+1} \\
& -\frac{g \Delta t}{2} \beta_{x x}\left(x_{i}^{n+1}\right) \delta x_{i}^{n+1}, \quad i=2, \ldots, N, \\
\delta p^{n+1}= & \delta p^{n+\frac{1}{2}}-\frac{1}{2} \nu_{1} \Delta t \delta q^{n+1}+\frac{3}{2} \nu_{2} \Delta t\left(q^{n+1}\right)^{2} \delta q^{n+1}, \\
= & \\
& \\
&
\end{aligned}
$$

where

$$
A_{i}^{n}=\frac{g \Delta a \Delta t}{2} \frac{\chi_{i}^{2}}{\left(x_{i+1}^{n}-x_{i}^{n}\right)^{3} \sigma\left(x_{i}^{n}\right)} \quad \text { and } \quad B_{i}^{n}=\frac{g \Delta a \Delta t}{4} \frac{\sigma_{x}\left(x_{i}^{n}\right) \chi_{i}^{2}}{\left(x_{i+1}^{n}-x_{i}^{n}\right)^{2} \sigma^{2}\left(x_{i}^{n}\right)} .
$$


The symplectic form is defined in (5.28). The numerical scheme is symplectic if

$$
\Omega_{N}^{n+1}=\Omega_{N}^{n} \quad \text { for all } n
$$

From (1.34) for the $(q, p)$ component of the symplectic form (5.28)

$$
\begin{aligned}
\delta p^{n+1} \wedge \delta q^{n+1} & =\left(\delta p^{n+\frac{1}{2}}-\frac{1}{2} \nu_{1} \Delta t \delta q^{n+1}+\frac{3}{2} \nu_{2} \Delta t\left(q^{n+1}\right)^{2} \delta q^{n+1}\right) \wedge \delta q^{n+1} \\
& =\delta p^{n+\frac{1}{2}} \wedge \delta q^{n+1} \\
& =\delta p^{n+\frac{1}{2}} \wedge\left(\delta q^{n}+\frac{\Delta t}{m_{v}} \delta p^{n+\frac{1}{2}}-\frac{\Delta t}{m_{v}} \delta \sigma_{N}^{n+\frac{1}{2}}\right) \\
& =\left(\delta p^{n}-\frac{1}{2} \nu_{1} \Delta t \delta q^{n}+\frac{3}{2} \nu_{2} \Delta t\left(q^{n}\right)^{2} \delta q^{n}\right) \wedge\left(\delta q^{n}-\frac{\Delta t}{m_{v}} \delta \sigma_{N}^{n+\frac{1}{2}}\right),
\end{aligned}
$$

and so

$$
\delta p^{n+1} \wedge \delta q^{n+1}=\delta p^{n} \wedge \delta q^{n}-\frac{\Delta t}{m_{v}} \delta p^{n} \wedge \delta \sigma_{N}^{n+\frac{1}{2}}+\frac{1}{2} \nu_{1} \frac{\Delta t^{2}}{m_{v}} \delta q^{n} \wedge \delta \sigma_{N}^{n+\frac{1}{2}}-\frac{3}{2} \nu_{2} \frac{\Delta t^{2}}{m_{v}}\left(q^{n}\right)^{2} \delta q^{n} \wedge \delta \sigma_{N}^{n+\frac{1}{2}}
$$

Also from (1.34) for the $(x, w)$ component of the symplectic form (5.28) and for $i=2, \ldots, N$,

$$
\begin{aligned}
\delta w_{i}^{n+1} \wedge \delta x_{i}^{n+1}= & \delta w_{i}^{n+1 / 2} \wedge \delta x_{i}^{n+1}+\frac{A_{i}^{n+1}}{\chi_{i}} \delta x_{i+1}^{n+1} \wedge \delta x_{i}^{n+1}+\frac{A_{i-1}^{n+1}}{\chi_{i}} \delta x_{i-1}^{n+1} \wedge \delta x_{i}^{n+1} \\
& -\frac{B_{i}^{n+1}}{\chi_{i}} \delta x_{i+1}^{n+1} \wedge \delta x_{i}^{n+1}-\frac{B_{i-1}^{n+1}}{\chi_{i}} \delta x_{i-1}^{n+1} \wedge \delta x_{i}^{n+1}
\end{aligned}
$$

But

$$
\begin{aligned}
\delta w_{i}^{n+1 / 2} \wedge \delta x_{i}^{n+1}= & \delta w_{i}^{n+1 / 2} \wedge\left(\delta x_{i}^{n}-\frac{\Delta t}{m_{v}} \delta p^{n+\frac{1}{2}}+\frac{\Delta t}{m_{v}} \delta \sigma_{N}^{n+\frac{1}{2}}\right) \\
= & \delta w_{i}^{n} \wedge \delta x_{i}^{n}+\frac{A_{i}^{n}}{\chi_{i}} \delta x_{i+1}^{n} \wedge \delta x_{i}^{n}+\frac{A_{i-1}^{n}}{\chi_{i}} \delta x_{i-1}^{n} \wedge \delta x_{i}^{n}-\frac{B_{i}^{n}}{\chi_{i}} \delta x_{i+1}^{n} \wedge \delta x_{i}^{n} \\
& -\frac{B_{i-1}^{n}}{\chi_{i}} \delta x_{i-1}^{n} \wedge \delta x_{i}^{n}-\frac{\Delta t}{m_{v}} \delta w_{i}^{n+1 / 2} \wedge \delta p^{n+1 / 2}+\frac{\Delta t}{m_{v}} \delta w_{i}^{n+1 / 2} \wedge \delta \sigma_{N}^{n+1 / 2} .
\end{aligned}
$$

Hence

$$
\begin{aligned}
\delta w_{i}^{n+1} \wedge \delta x_{i}^{n+1}= & \delta w_{i}^{n+1 / 2} \wedge\left(\delta x_{i}^{n}-\frac{\Delta t}{m_{v}} \delta p^{n+\frac{1}{2}}+\frac{\Delta t}{m_{v}} \delta \sigma_{N}^{n+\frac{1}{2}}\right) \\
= & \delta w_{i}^{n} \wedge \delta x_{i}^{n}+\frac{A_{i}^{n}}{\chi_{i}} \delta x_{i+1}^{n} \wedge \delta x_{i}^{n}+\frac{A_{i-1}^{n}}{\chi_{i}} \delta x_{i-1}^{n} \wedge \delta x_{i}^{n}-\frac{B_{i}^{n}}{\chi_{i}} \delta x_{i+1}^{n} \wedge \delta x_{i}^{n} \\
& -\frac{B_{i-1}^{n}}{\chi_{i}} \delta x_{i-1}^{n} \wedge \delta x_{i}^{n}-\frac{\Delta t}{m_{v}} \delta w_{i}^{n+1 / 2} \wedge \delta p^{n+1 / 2}+\frac{\Delta t}{m_{v}} \delta w_{i}^{n+1 / 2} \wedge \delta \sigma_{N}^{n+1 / 2} \\
& +\frac{A_{i}^{n+1}}{\chi_{i}} \delta x_{i+1}^{n+1} \wedge \delta x_{i}^{n+1}+\frac{A_{i-1}^{n+1}}{\chi_{i}} \delta x_{i-1}^{n+1} \wedge \delta x_{i}^{n+1}-\frac{B_{i}^{n+1}}{\chi_{i}} \delta x_{i+1}^{n+1} \wedge \delta x_{i}^{n+1} \\
& -\frac{B_{i-1}^{n+1}}{\chi_{i}} \delta x_{i-1}^{n+1} \wedge \delta x_{i}^{n+1} .
\end{aligned}
$$


Before summing to obtain the symplectic form, first it is shown that the $A_{i}^{n}$ and $B_{i}^{n}$ terms vanish when summed. First multiply (1.37) by $\chi_{i} \Delta a$ then from the definition,

$$
\sum_{i=2}^{N} A_{i}^{n} \delta x_{i+1}^{n} \wedge \delta x_{i}^{n} \Delta a=\sum_{i=2}^{N-1} A_{i}^{n} \delta x_{i+1}^{n} \wedge \delta x_{i}^{n} \Delta a=\sum_{i=3}^{N} A_{i-1}^{n} \delta x_{i}^{n} \wedge \delta x_{i-1}^{n} \Delta a,
$$

using the fact that $\delta x_{N+1}^{n}=0$, and shifting the index by one in the second equality. Similarly,

$$
\sum_{i=2}^{N} A_{i-1}^{n} \delta x_{i-1}^{n} \wedge \delta x_{i}^{n} \Delta a=\sum_{i=3}^{N} A_{i-1}^{n} \delta x_{i-1}^{n} \wedge \delta x_{i}^{n} \Delta a=-\sum_{i=3}^{N} A_{i-1}^{n} \delta x_{i}^{n} \wedge \delta x_{i-1}^{n} \Delta a,
$$

using the fact that $\delta x_{1}^{n}=0$ and skew-symmetry of wedge. Hence

$$
\sum_{i=2}^{N} A_{i}^{n} \delta x_{i+1}^{n} \wedge \delta x_{i}^{n} \Delta a+\sum_{i=2}^{N} A_{i-1}^{n} \delta x_{i-1}^{n} \wedge \delta x_{i}^{n} \Delta a=0 .
$$

A similar argument proves that

$$
\sum_{i=2}^{N} A_{i}^{n+1} \delta x_{i+1}^{n+1} \wedge \delta x_{i}^{n+1} \Delta a+\sum_{i=2}^{N} A_{i-1}^{n+1} \delta x_{i-1}^{n+1} \wedge \delta x_{i}^{n+1} \Delta a=0 .
$$

Also for the $B_{i}^{n}$ terms

$$
\sum_{i=2}^{N}-B_{i-1}^{n} \delta x_{i-1}^{n} \wedge \delta x_{i}^{n} \Delta a=\sum_{i=3}^{N}-B_{i-1}^{n} \delta x_{i-1}^{n} \wedge \delta x_{i}^{n} \Delta a=\sum_{i=3}^{N} B_{i-1}^{n} \delta x_{i}^{n} \wedge \delta x_{i-1}^{n} \Delta a,
$$

and

$$
\sum_{i=2}^{N}-B_{i}^{n} \delta x_{i+1}^{n} \wedge \delta x_{i}^{n} \Delta a=\sum_{i=2}^{N-1}-B_{i}^{n} \delta x_{i+1}^{n} \wedge \delta x_{i}^{n} \Delta a=\sum_{i=3}^{N}-B_{i-1}^{n} \delta x_{i}^{n} \wedge \delta x_{i-1}^{n} \Delta a
$$

and hence

$$
\sum_{i=2}^{N}-B_{i}^{n} \delta x_{i+1}^{n} \wedge \delta x_{i}^{n} \Delta a-\sum_{i=2}^{N} B_{i-1}^{n} \delta x_{i-1}^{n} \wedge \delta x_{i}^{n} \Delta a=0 .
$$

A similar argument proves that

$$
\sum_{i=2}^{N}-B_{i}^{n+1} \delta x_{i+1}^{n+1} \wedge \delta x_{i}^{n+1} \Delta a-\sum_{i=2}^{N} B_{i-1}^{n+1} \delta x_{i-1}^{n+1} \wedge \delta x_{i}^{n+1} \Delta a=0 .
$$

Summing the $(x, w)$ and $(q, p)$ components of the symplectic form and using (1.38), (1.39), (1.40), and (1.41) leads to:

$$
\begin{aligned}
\Omega_{N}^{n+1}= & \Omega_{N}^{n}-\frac{\Delta t}{m_{v}}\left(\sum_{i=2}^{N} \delta w_{i}^{n+1 / 2} \rho \chi_{i} \Delta a\right) \wedge \delta p^{n+1 / 2}+\frac{\Delta t}{m_{v}}\left(\sum_{i=2}^{N} \delta w_{i}^{n+1 / 2} \rho \chi_{i} \Delta a\right) \wedge \delta \sigma_{N}^{n+1 / 2} \\
& -\frac{\Delta t}{m_{v}} \delta p^{n} \wedge \delta \sigma_{N}^{n+\frac{1}{2}}+\frac{1}{2} \nu_{1} \frac{\Delta t^{2}}{m_{v}} \delta q^{n} \wedge \delta \sigma_{N}^{n+\frac{1}{2}}-\frac{3}{2} \nu_{2} \frac{\Delta t^{2}}{m_{v}}\left(q^{n}\right)^{2} \delta q^{n} \wedge \delta \sigma_{N}^{n+\frac{1}{2}}
\end{aligned}
$$


But note that

$$
\sum_{i=2}^{N} \delta w_{i}^{n+1 / 2} \rho \chi_{i} \Delta a=\delta \sigma_{N}^{n+\frac{1}{2}}-\frac{1}{2} \rho\left(\chi_{1}+\chi_{N+1}\right) \Delta a \delta w_{1}^{n+\frac{1}{2}},
$$

and also from the boundary condition (5.27)

$$
\delta w_{1}^{n+1 / 2}=\frac{1}{m_{v}} \delta p^{n+1 / 2}-\frac{1}{m_{v}} \delta \sigma_{N}^{n+1 / 2} .
$$

Now substitution of (1.43) and (1.44) into (1.42) simplifies this expression to (1.35).

\section{References}

[1] B.H. ADEE \& I. CAGLAYAN. The effects of free water on deck on the motions and stability of vessels, In: Proceedings of Second International Conference on the Stability of Ships and Ocean Vehicles. SNAME, Springer, Tokyo (1982).

[2] H. Alemi Ardakani. Rigid-Body Motion with Interior Shallow-Water Sloshing, Ph.D. Thesis, University of Surrey, UK (2010).

[3] H. Alemi ArdakAni \& T.J. BRIDges. Dynamic coupling between shallow-water sloshing and horizontal vehicle motion, European Journal of Applied Mathematics 21 479-517 (2010).

[4] H. Alemi Ardakani, T.J. Bridges \& M.R Turner. Resonance in a model for Cooker's sloshing experiment, European Journal of Mechanics B/Fluids 36 25-38 (2012).

[5] H. Alemi Ardakani, T.J. Bridges \& M.R Turner. Shallow-water sloshing in a moving vessel with variable cross-section and wetting-drying using an extension of George's well-balanced finite volume solver, Preprint (2015).

[6] I. CAGLAYAN \& R.L. STORCH. Stability of fishing vessels with water on deck: a review, J. Ship Research 26 106-116 (1982).

[7] W. Chester. Resonant oscillations of water waves. I. Theory, Proc. Royal Soc. London A 306 5-22 (1968).

[8] M.J. COOKER. Water waves in a suspended container, Wave Motion 20, 385-395 (1994).

[9] E.A. Cox, J.P. Gleeson \& M.P. MoRTEll. Nonlinear sloshing and passage through resonance in a shallow water tank, Z. angew. Math. Phys. 56 645-680 (2005).

[10] J. Dillingham. Motion studies of a vessel with water on deck, Wave Motion 18 38-50 (1981).

[11] F. Dubois, N. PetiT \& P. Rouchon. Motion planning and nonlinear simulations for a tank containing fluid, European Control Conference, Karlsruhe, 6 pages (1999).

[12] O.M. Faltinsen \& A. Timokna. Sloshing, Second Edition, Cambridge University Press (2014). 
[13] J.T. Feddema, C.R. Dohrmann, G.G. Parker, R.D. Robinett, V.J. Romero \& D.J. SCHMITT. Control for slosh-free motion of an open container, IEEE Control Systems Magazine 17 29-36 (1997).

[14] J.B. FRANDSEN. Numerical predictions of tuned liquid tank structural systems, Journal of Fluids and Structures 20 309-329 (2005).

[15] P. Garcia-Navarro \& M.E. Vazquez-Cendon. On numerical treatment of the source terms in the shallow water equations, Computers \& Fluids 29 951-979 (2000).

[16] P. GLAISTER. Approximate Riemann solutions of the shallow water equations, Journal of Hydraulic Research 26 293-306 (1988).

[17] M. GRUndelius \& B. Bernhardsson. Control of liquid slosh in an industrial packaging machine, IEEE Int. Conf. Control Appl., Kohala Coast, Hawaii, 6 pages (1999).

[18] E. Hairer, C. Lubich \& G.WAnNER. Geometric numerical integration illustrated by the Störmer-Verlet method, Acta Numerica 12 399-450 (2003).

[19] G. HernandeZ-Duenas \& S. KARni. Shallow water flows in channels, J. Sci. Comput. 48 190-208 (2011).

[20] R. A. Ibrahim Liquid Sloshing Dynamics, Cambridge University Press (2005).

[21] T. IKEDA \& N. NAKAGAWA. Non-linear vibrations of a structure caused by water sloshing in a rectangular tank, Journal of Sound and Vibration $20123-41$ (1997).

[22] A.F. Jones \& A. Hulme. The hydrodynamics of water on deck, J. Ship Research 31 125-135 (1987).

[23] B. Leimkuhler \& S. ReICH. Simulating Hamiltonian Dynamics, Cambridge University Press (2004).

[24] B. VAN LEER. On the relation between the upwind-differencing schemes of Godunov, Engquist-Osher and Roe, SIAM J. Sci. Statist. Comput. 5 1-20 (1984).

[25] J.E. MARSDen \& M. West. Discrete mechanics and variational integrators, Acta Numerica $10357-514$ (2001).

[26] N.N. Moiseyev \& V.V. Rumyantsev. Dynamic Stability of Bodies Containing Fluid, Springer (1968).

[27] J. R. OCKENDON \& H. OCKENDON. Resonant surface waves, J. Fluid Mech. 59 397-413 (1973).

[28] H. Ockendon, J. R. Ockendon \& A. D. Johnson. Resonant sloshing in shallow water, J. Fluid Mech. 167 465-479 (1986).

[29] C. PRIEUR \& J. DE HAlleuX. Stabilization of a 1-D tank containing a fluid modeled by the shallow water equations, Systems \& Control Letters 52, 167-178 (2004).

[30] P.L. RoE. Approximate Riemann solvers, parameter vectors, and difference schemes, Journal of Computational Physics 43 357-372 (1981). 
[31] P.L. RoE. Upwind differencing schemes for hyperbolic conservation laws with source terms, In: Nonlinear Hyperbolic Problems. Proc. Adv. Res. Workshop, St. Étienne, 1986. Lect. Notes Math. Springer, Berlin 1270 41-51 (1987).

[32] M.R Turner, H. Alemi ARdakAni \& T.J. BRIDges. Instability of sloshing motion in a vessel undergoing pivoted oscillations, Journal of Fluids and Structures 52 166-180 (2015).

[33] M.R TURNeR \& T.J. BRIDgeS. Nonlinear energy transfer between fluid sloshing and vessel motion, J. Fluid Mech. 719 606-636 (2013).

[34] M.R Turner, T.J. Bridges \& H. Alemi Ardakani. Dynamic coupling in Cooker's sloshing experiment with baffles, Physics of Fluids 25-112102 1-22 (2013).

[35] M.R Turner, T.J. BRidges \& H. Alemi ArdakAni. The pendulum-slosh problem: simulation using a time-dependent conformal mapping, Journal of Fluids and Structures 59 202-223 (2015).

[36] M.P. Tzamtzi \& N.D. KouvaKas. Sloshing control in tilting phases of the pouring process, Inter. J. Math., Phys. Eng. Sciences 1 175-182 (2007).

[37] M.P. Tzamtzi \& N.D. KouvaKas. Robustness of a robot control scheme for liquid transfer, in Novel Algorithms and Techniques in Telecommunications, Automation and Industrial Electronics, Edited by T. SoBH ET AL., Springer-Verlag 154-161 (2008).

[38] M.E. VAZQUEZ-CENDON. Improved treatment of source terms in upwind schemes for the shallow water equations in channels with irregular geometry, Journal of Computational Physics 148 497-526 (1999).

[39] J. H. G. Verhagen \& L. VAn WijngaArden. Non-linear oscillations of fluid in a container, J. Fluid Mech. 22 737-751 (1965).

[40] P.D. WEIDMAN. Synchronous sloshing in free and suspended containers, in APS Division of Fluid Dynamics, 47th Annual Meeting, Atlanta, GA, 20-22 November (1994).

[41] P.D. WeIDMAN. Sloshing in suspended containers, in APS Division of Fluid Dynamics, 58th Annual Meeting, Chicago, IL, 20-22 November (2005).

[42] S. AUS DER WIESCHE. Computational slosh dynamics: theory and industrial application, Comp. Mech. 30 374-387 (2003).

[43] J. YU. Effects of finite water depth on natural frequencies of suspended water tanks, Stud. Appl. Math. 125 373-391 (2010). 